\title{
Computational fluid dynamics investigation of the novel hybrid comprehensive stage II operation
}

Marwan Hameed, PhD, ${ }^{\mathrm{a}, \mathrm{b}}$ Ray Prather, PhD, ${ }^{\mathrm{a}, \mathrm{b}, \mathrm{c}}$ Eduardo Divo, PhD, ${ }^{\mathrm{b}}$ Alain Kassab, PhD, ${ }^{\mathrm{a}}$ David Nykanen, MD, ${ }^{\mathrm{c}}$ Michael Farias, MD, MS, MBA, ${ }^{\mathrm{c}}$ and William M. DeCampli, MD, PhD ${ }^{\mathrm{a}, \mathrm{c}, \mathrm{d}}$

\section{ABSTRACT}

Background: The hybrid comprehensive stage $2\left(\mathrm{HCS}_{2}\right)$ procedure is a novel palliative operation applicable to a select subset of single ventricle patients with adequate native antegrade aortic flow to the upper body. Flow to the descending aorta, through the pulmonary outlet and ductal arch, is influenced by a stented intrapulmonary baffle connecting the branch pulmonary arteries. We used computational fluid dynamics (CFD) to elucidate the hemodynamic characteristics of this reconstruction.

Methods: We used multiscale CFD analysis of a synthetic, patient-derived HCS2 anatomic configuration with unsteady laminar flow conditions and a nonNewtonian blood model to quantify the resultant hemodynamics. The 3-dimensional CFD model was coupled to a o-dimensional lumped parameter model of the peripheral circulation to determine the required boundary conditions.

Results: For the specific anatomy studied, the intrapulmonary baffle did not obstruct flow from the pulmonary trunk to ductal arch as long as the distance between the anterior pulmonary artery wall and baffle wall exceeded $\sim 7 \mathrm{~mm}$. Vortex shedding off of the baffle wall did not develop, because of the short distance to the ductal arch. The stented baffle experienced significantly uneven "inward" loading from the systemic side. Pulmonary outlet flow separation distal to the baffle produced a low-speed recirculation region.

Conclusions: Hemodynamic patterns in this complex anatomy are generally favorable. Low flow recirculation could be mitigated by preoperative shape optimization. Calculated inward stresses on the pulmonary baffle can be used in the future to study baffle stent deformation, which is expected to be small. (JTCVS Open 2021;7:308-23)

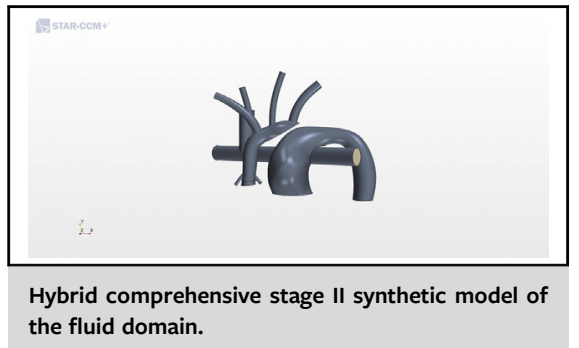

CENTRAL MESSAGE

Hemodynamic patterns in the hybrid comprehensive stage 2 anatomy are generally favorable. Low flow recirculation could be mitigated by preoperative shape optimization.

\section{PERSPECTIVE}

The hybrid comprehensive stage 2 is a novel palliative operation applicable to a subset of single ventricle patients with adequate native antegrade aortic flow. The flow is influenced by a stented intrapulmonary baffle connecting the branch pulmonary arteries. Computational fluid dynamics elucidated the hemodynamic characteristics of this reconstruction and determined them to be generally favorable.

See Commentaries on pages 324 and 325 .
From the ${ }^{\mathrm{a}}$ Department of Mechanical and Aerospace Engineering, and ${ }^{\mathrm{d}}$ Department of Clinical Sciences, College of Medicine, University of Central Florida, Orlando, Fla; ${ }^{\mathrm{b}}$ Department of Mechanical Engineering, Embry-Riddle Aeronautical University, Daytona Beach, Fla; and ${ }^{\mathrm{C} P e d i a t r i c}$ Cardiology, Arnold Palmer Hospital for Children, Orlando, Fla.

This study was supported by an American Heart Association Innovative Award (AHA 17GRNT33411154).

Received for publication April 15, 2021; accepted for publication April 19, 2021; available ahead of print June 10, 2021

Address for reprints: William M. DeCampli, MD, PhD, The Heart Center, Arnold Palmer Hospital for Children, 92 W Miller St, MP 307, Orlando, FL 32806 (E-mail: William.decampli@orlandohealth.com).

2666-2736

Copyright (c) 2021 The Author(s). Published by Elsevier Inc. on behalf of The American Association for Thoracic Surgery. This is an open access article under the CC BY-NC-ND license (http://creativecommons.org/licenses/by-nc-nd/4.0/).

https://doi.org/10.1016/j.xjon.2021.04.009
Single ventricle (SV) anomalies constitute some of the most severe forms of congenital heart disease (CHD). The associated mortality is high, with some studies showing only $60 \%-70 \%$ survival past 5 years of age. ${ }^{1,2}$ Most of the surviving children have a life-long decrease in exercise capacity, and more than one-half experience developmental delays. ${ }^{3,4}$ A 3-stage surgical approach has been widely adopted to treat most variants of SV CHD, including hypoplastic left heart syndrome (HLHS). The conventional approach includes the stage 1 (Norwood) operation, a major procedure necessitating cardiopulmonary bypass commonly performed before 1 week of age. ${ }^{5}$ The period around the stage 1 operation has been identified as the time of greatest risk for mortality and morbidity., 


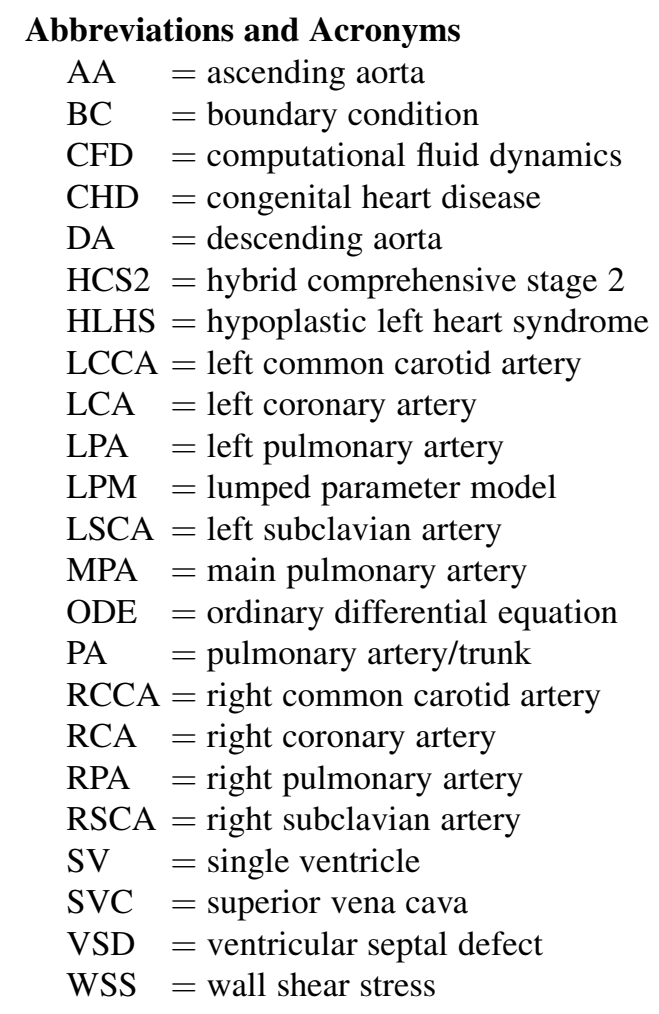

The "hybrid" sequence of staged operations is an alternative to the conventional approach. The hybrid stage 1 avoids cardiopulmonary bypass in the neonatal period and reduces neonatal morbidity. ${ }^{8-10}$ In some centers, morbidity and mortality in the subsequently required comprehensive stage 2 operation is greater than that of the traditional stage 2 operation. ${ }^{11,12}$

A decrease in the complexity of the second-stage hybrid palliation can potentially lead to decreased morbidity and mortality. To reduce the total trauma to the patient, we previously proposed an alternative hybrid technique to the standard comprehensive stage 2 operation (HCS2) that is applicable to the subset of SV patients with sufficient antegrade aortic flow to sustain upper body perfusion. ${ }^{13,14}$ Congenital cardiac anomalies may include an unbalanced atrioventricular canal, double-inlet left ventricle, tricuspid atresia with ventriculoarterial discordance, mitral atresia with a large ventricular septal defect (VSD), and some cases of HLHS with mitral and aortic stenosis. The technique avoids the creation of a Damus-KayeStansel connection and surgical arch reconstruction by maintaining ductal patency.

The procedure includes branch pulmonary artery debanding and a limited incision into the main pulmonary artery (MPA). Within the MPA ("neoaorta"), a stent is placed to reinforce a baffle that bridges the ostia of the branch pulmonary arteries. ${ }^{13}$ The previously stented "ductal arch" is dilated and restented. A bidirectional superior cavopulmonary connection completes the operation. As a result, systemic blood flow is partitioned into direct aortic outflow to the upper body and "neoaortic" (pulmonary trunk) flow through the ductal arch to the lower body.

The anatomy of the HCS2 reconstruction has a unique configuration, with pulsatile flow under systemic pressure traveling over the pulmonary artery baffle with a semicircular shape. As such, there is concern that the intrapulmonary baffle may obstruct systemic flow and result in excessive vortex formation and recirculation zones in the main pulmonary artery. Furthermore, the stent itself is subject to a net inward stress from the systemic circulation. Multiscale CFD studies have been successfully used to explore adaptations of the Norwood procedure and other stages of SV surgical palliation. ${ }^{15-22}$ Such an approach is ideal for elucidating the characteristics of flow in the HCS2 reconstruction. In this study, we used multiscale computational fluid dynamics (CFD) to determine the conditions under which obstruction, vortex formation, and recirculation zones may occur with the HCS2 reconstruction.

\section{METHODS \\ Geometry}

A synthetic 3-dimensional model was built using SolidWorks (Dassault Systèmes, Vélizy-Villacoublay, France) that included the main pulmonary artery, branch pulmonary arteries, descending aorta, ascending aorta, coronary arteries, carotid arteries, subclavian arteries, and superior vena cava (SVC). Deidentified angiographic images of a 7-month-old infant who underwent the HCS2 procedure were used to determine the dimensions of various vessels for construction of the synthetic model (Figure 1, B). The idealized model in Figure 1, $A$ was modified for 6 variations of MPA diameters to study pressure drop across the baffle in the systemic region.

\section{Fluid Domain Solver}

The hemodynamic analysis was carried out with commercial CFD software (StarCCM+; Siemens Digital Industries, Plano, Tex), which provides an integrated meshing and flow solver environment. Results are obtained by numerically solving the time-resolved conservation equations of mass and linear momentum:

$$
\begin{gathered}
\nabla \cdot \vec{V}=0 \\
\rho\left(\frac{\partial \vec{V}}{\partial t}+\vec{V} \cdot \nabla \vec{V}\right)=-\nabla p+\nabla \cdot \underline{\underline{\sigma}}
\end{gathered}
$$

where $\rho$ is the density of blood, $1060 \mathrm{~kg} / \mathrm{m}^{3} ; \vec{V}$ is the velocity; $p$ is the pressure; and $\underline{\underline{\sigma}}=\mu(\dot{\gamma})\left[\nabla \vec{V}+(\nabla \vec{V})^{T}\right]$ is the viscous stress tensor, with a given relation for the viscosity $\mu(\dot{\gamma})$ as a function of shear rate, $(\dot{\gamma})$. The non-Newtonian rheology of blood is modeled after the Carreau-Yasuda model for viscosity as

$$
\mu(\dot{\gamma})=\mu_{\infty}+\left(\mu_{0}-\mu_{\infty}\right) \frac{1}{\left[1+(\lambda \dot{\gamma})^{2}\right]^{\frac{1}{3}}}
$$




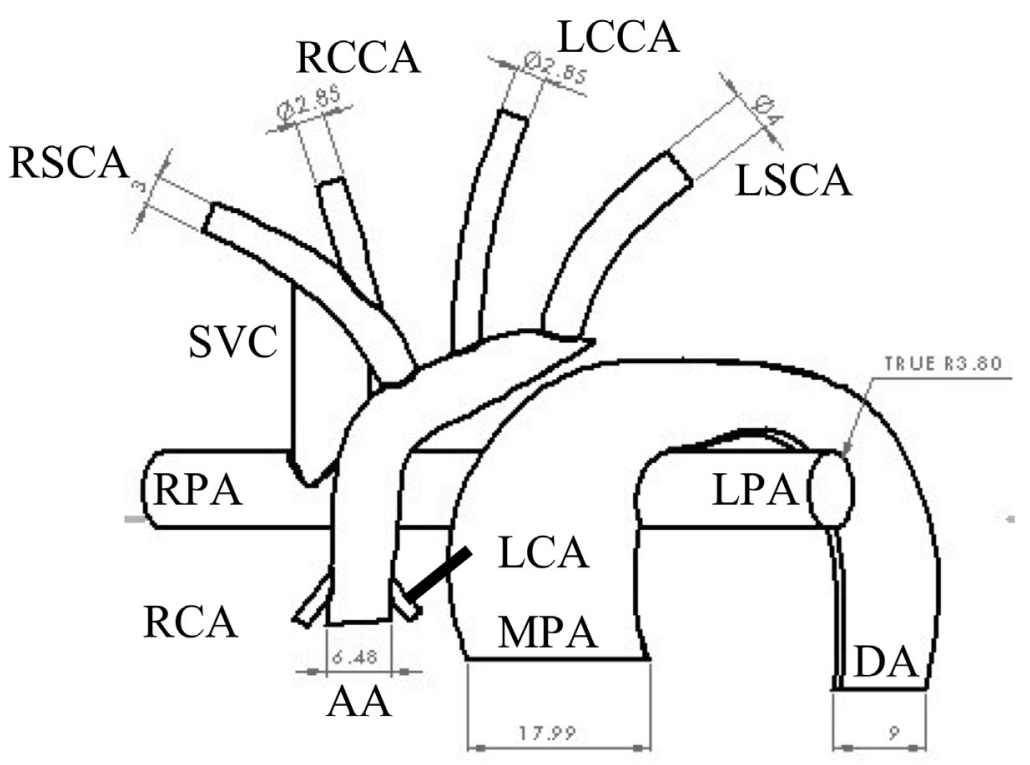

A

B
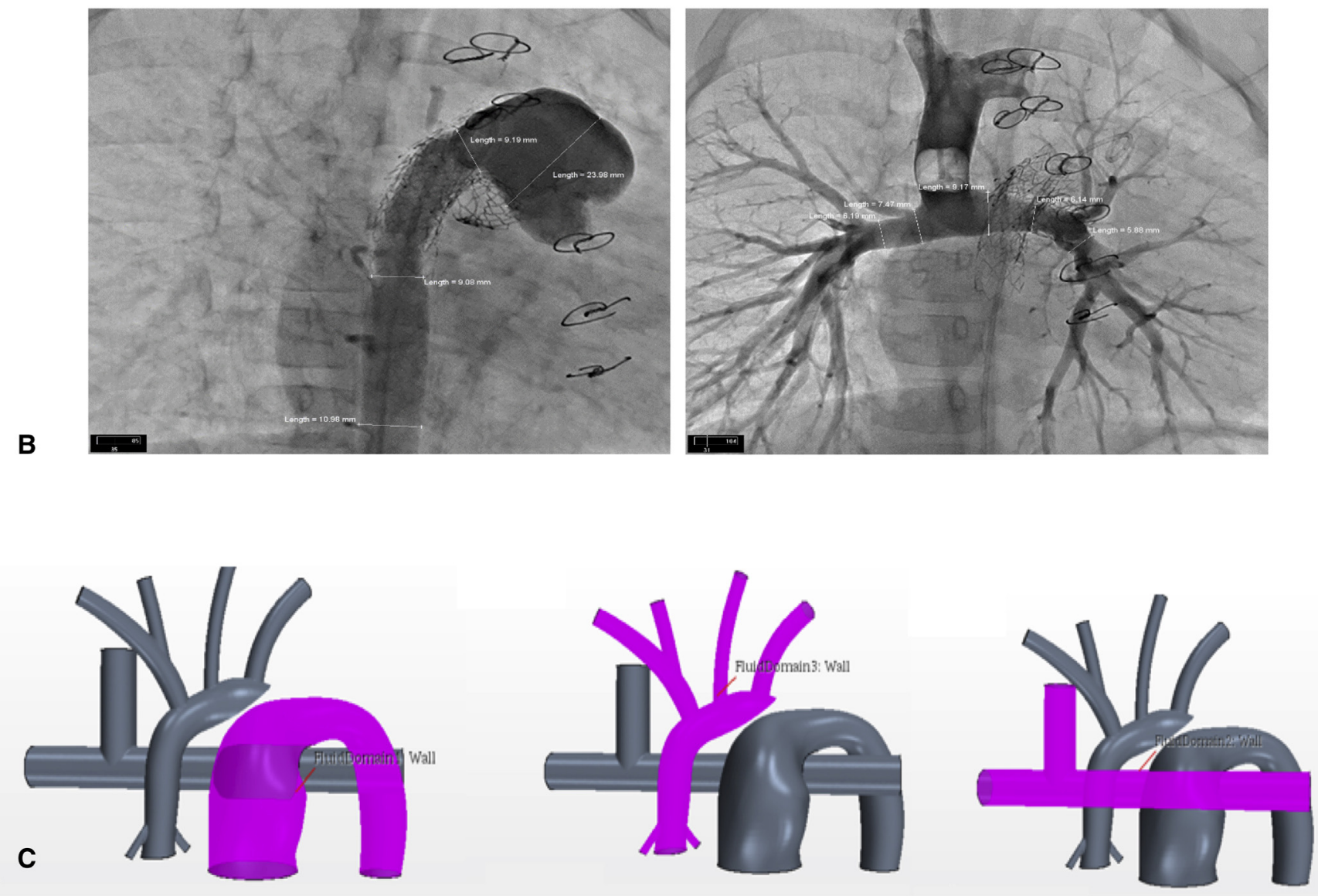

FIGURE 1. A, Dimensions of the synthetic model with diameters of labeled branches (in millimeters). B, Deidentified X-ray angiography for the lower body systemic flow (left) and pulmonary flow (right) postoperatively. C, The 3 separate fluid regions: lower systemic flow (left), upper systemic flow (mid$d l e$ ), and pulmonary flow (right). RCCA, Right common carotid artery; LCCA, left common carotid artery; RSCA, right subclavian artery; LSCA, left subclavian artery; $S V C$, superior vena cava; $R P A$, right pulmonary artery; $L P A$, left pulmonary artery; $L C A$, left coronary artery; $R C A$, right coronary artery; $M P A$, main pulmonary artery; $A A$, ascending aorta; $D A$, descending aorta. 
where $\mu_{0}$ is the zero-shear viscosity, $\mu_{\infty}$ is the infinite-shear viscosity, $\lambda$ is the relaxation time constant, and is the shear rate. The blood model constants for a hematocrit of $40 \%$ were obtained by curve fit applied to documented clinical data. ${ }^{23}$

On importing the geometry, 3 separate fluid domains were maintained: (1) the SVC flow to the pulmonary arteries, (2) the systemic flow originating from the pulmonary root to the lower body, and (3) the systemic flow originating in the aortic root to the upper body (Figure 1, C).

The domains are discretized in the destination software using an unstructured tetrahedral mesh with a near-wall, 3-prism layer refinement, which generated approximately 1.3 million cells following a grid independence study. Following the mesh convergence study, we estimated the Reynolds number $(\mathrm{Re})$ to determine the flow modeling assumption. We found the maximum to be $\operatorname{Re}=1807$, which is well within the laminar flow regime.

\section{Lumped Parameter Model}

A total of 12 pulsatile boundary conditions (BCs) were applied to the domain inlets and outlets (pressure and mass flow rate). These timedependent BCs were obtained from a lumped parameter model (LPM) of the peripheral circulation coupled to the CFD represented by the circuit schematic in Figure 2, A. This circuit models a patient with mitral atresia, a large VSD, a moderately underdeveloped left ventricle, a functioning aortic valve, and a hypoplastic arch providing adequate upper body perfusion.

In Figure 2, $A$, the resistor labeled "R_da_lsa" can be tuned to obtain any desired level of distal arch stenosis. The current model assumes complete distal arch obstruction; thus, this resistor is tuned to a 100-fold greater magnitude compared with that of the neighboring resistors. No VSD is directly present in the CFD domain; however, "R_aorta" expresses a flow-dependent nonlinear resistance, typical of an orifice.

The pressure drop across the circuit segment containing the septal defect can be represented by $\Delta P(t)=C \dot{Q}(t)^{2}$, which leads to a nonlinear resistance in terms of volumetric flow rate, $R(\dot{Q})=C \dot{Q}(t){ }^{24-29}$ The Bernoulli equation for an orifice (Eq A.1) can be rewritten to determine an expression for resistance to be included in the LPM equations as

$$
R_{V S D}(\dot{Q})=\frac{\dot{Q} \rho}{266.64\left(1000^{2} K A_{V S D}\right)^{2}}
$$

Further manipulation of this expression detailed in Appendix E1, with the addition of a linear resistor to represent a portion of the aortic root in conjunction with the atrophied left ventricle traversed by flow, gives

$$
R_{\text {aorta }}(\dot{Q})=R_{V S D}+\frac{\dot{Q} \rho}{266.64\left(1000^{2} K A_{V S D}\right)^{2}}
$$

$R_{V S D}$ is tuned to yield proper pressure and mass flow rate BCs to the CFD.

The infant is assumed to have a heart rate of $120 \mathrm{bpm}$. The LPM allows us to derive 34 first-order ordinary differential equations defining 34 degrees of freedom solved using an in-house fourth-order adaptive timestepping Runge-Kutta solver. The LPM models vessel compliance as a capacitor (C), viscous drag as a resistor (R), flow inertia as an inductor (L), and the heart valves as diodes (D). ${ }^{30}$ A time-varying compliance abiding by an elastance function acts as a pump driving the flow in the LPM. $^{31}$

The LPM was tuned to produce a cardiac output for the nominal model of approximately $3 \mathrm{~L} /$ minute with cardiac cycle of 0.5 seconds. Owing to the presence of a VSD, the HCS2 has a nonnegligible aortic root flow output. Our LPM is tuned to have a $60 \%-40 \%$ outflow split between the MPA feeding the lower circulation and the ascending aorta supplying the upper circulation, respectively. Figure 3, $A$ displays a sample all the $\mathrm{BC}$ waveforms applied to our model.

Finally, we tuned the circuit to obtain a $70 \%-30 \%$ split between the left and the right coronary arteries. ${ }^{32,33}$

Reference mass flow data were obtained from magnetic resonance imaging studies, and pressure data were gathered from invasive cardiac catheterizations. Figure 3, $B$ displays deidentified sample catheter pressure tracings used to tune the LPM. The pressure waveform measured for the main pulmonary artery can be directly compared with the computed pressure waveform in Figure 3, A, showing similar features and providing a degree of validation.

\section{Coupling}

The LPM and the CFD domain are loosely coupled at the heart cycle level. ${ }^{30}$ This coupling allows for iterative updating of the respective CFD conduit resistances in the LPM. The CFD domain enables the user to measure pressure and mass flow rates at various intervals across the geometry, corresponding to the 0 -dimensional circuit nodes (pressure) and segments (mass flow). These measurements are first surface-averaged over the sample cross-section and subsequently time-averaged over the cycle. The resulting pressure and mass flow quantities are used to compute each vessel resistance based on Ohm's law (ratio of pressure across the segment and the mass flow across it), which are then updated in the LPM. Once the LPM solver converges, the resulting time-dependent waveforms can be imposed as BCs to the CFD. The initial tuning of the LPM represents an educated guess; thus, the aim of this iterative process is to obtain consistent $\mathrm{BCs}$. The resolved flow field is considered converged once both the $\mathrm{BC}$ and the resistance scalar value no longer change. Each iterative run in the multiscale coupling was carried out for a total of 3 heart cycles.

\section{RESULTS}

The HCS2 has a unique geometry in which the baffle presents a solid obstacle for the high-pressure lower body circulation originating from the right ventricle, resulting in unique flow patterns, such as flow obstruction and potential vortex formation.

\section{Systemic Pressure Drop}

Varying the minimum distance $(\mathrm{H})$ between the anterior MPA and the anterior baffle surface resulted in different pressure gradients across the pulmonary outflow tract and the ductal arch (Figure 4). Values are computed as surface-averaged and time-averaged pressures over a sampling surface, whereas peak values are the maximum values observed across the sampling surface throughout a single heart cycle.

The purpose of these pressure measurements was to ensure that any obstruction would not exceed a clinically accepted pressure drop of $10 \mathrm{~mm} \mathrm{Hg}$. Figure $4, B$ shows the pressure gradient, $\Delta \mathrm{p}=\mathrm{p}_{\mathrm{A}}-\mathrm{p}_{\mathrm{B}}$, versus $H$. Similar results were obtained for the pressure difference proximal to the baffle and the distal ductal arch.

The peak, $\Delta \mathrm{p}$, is strongly affected by an incremental obstruction of the flow (as $H$ decreases), while the surface averaged $\Delta \mathrm{p}$ experiences little change. The largest 


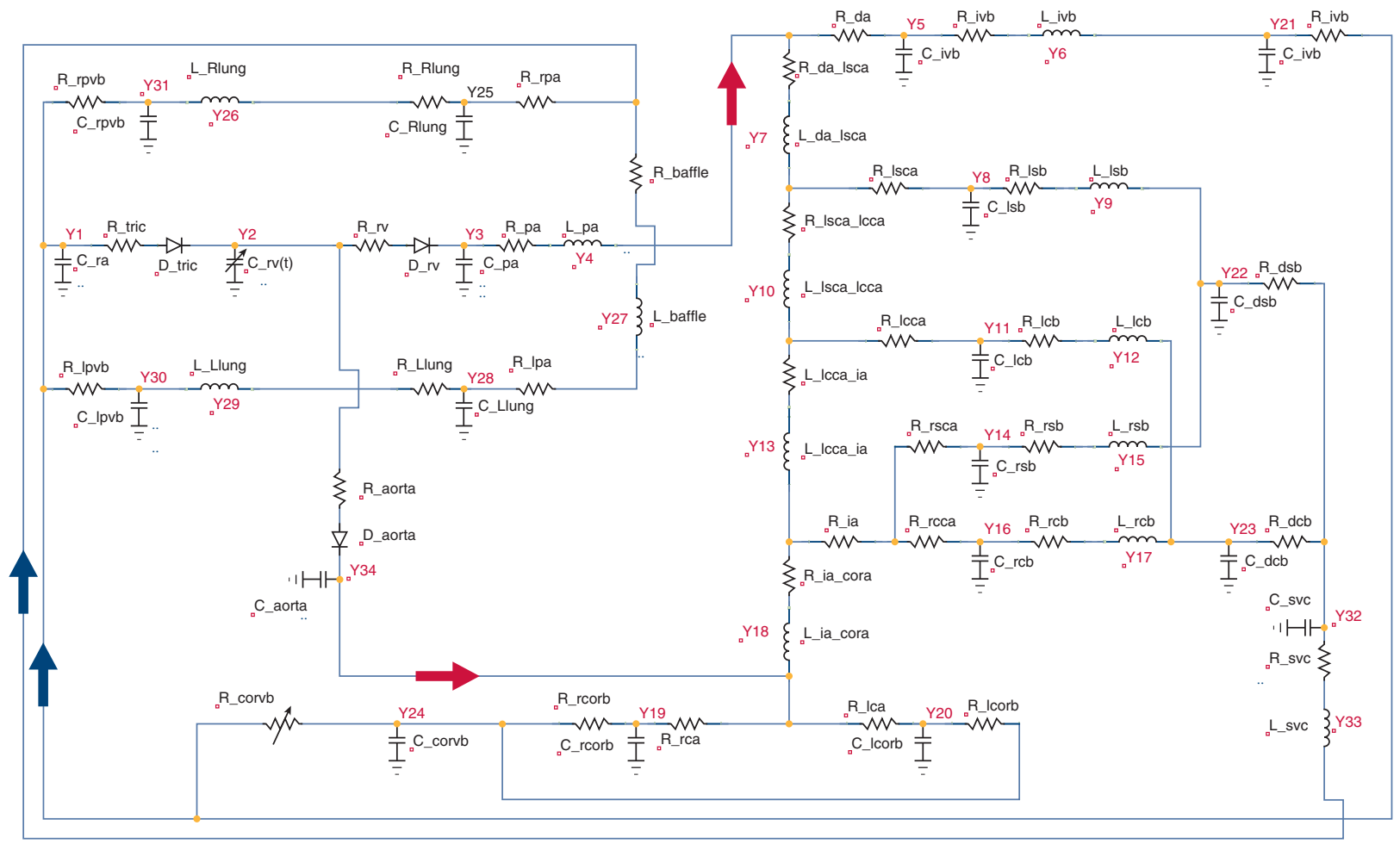

A
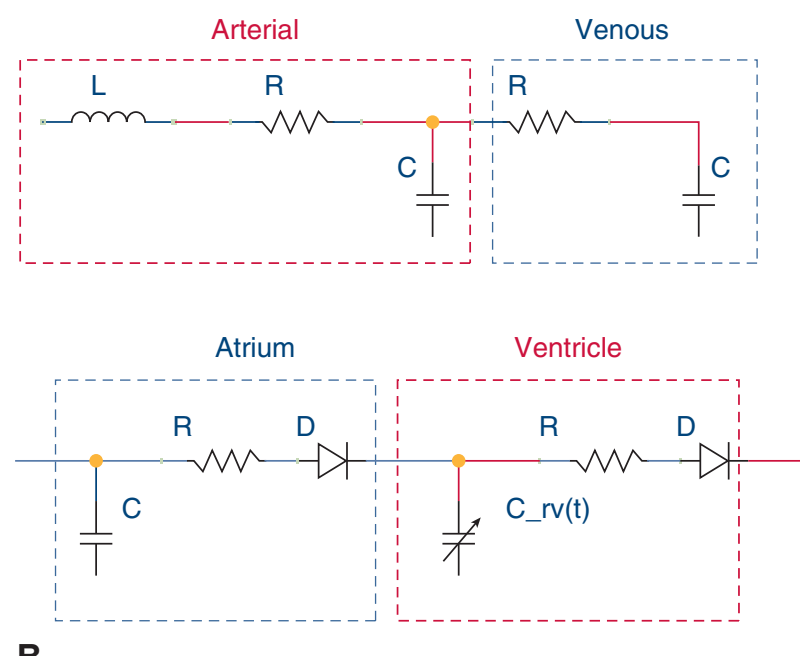

B

FIGURE 2. Lumped parameter model vascular circuit (A) and schematic of basic circuit elements (B) of venous and arterial beds (top) and heart chambers (bottom).

pressure drop localized to the area anterior to the baffle was $2.96 \mathrm{~mm} \mathrm{Hg}$, with a total gradient of $12.15 \mathrm{~mm} \mathrm{Hg}$ across the entire pulmonary artery and ductal arch. The largest pressure drop measured is $14.95 \mathrm{~mm} \mathrm{Hg}$, which, interestingly, was not associated with the smallest distance (value of $H$ ). When accounting for the distal arch contribution, the peak pressure difference counterintuitively shows a mild upward slope, indicating that for increasing MPA diameter, a slightly increased pressure drop is observed.
This can be explained by how the surgeon may enlarge the pulmonary trunk by placing a patch. This patch increases the volume of the pulmonary trunk. For a rigid wall model, this additional volume creates a region that acts similar to a reservoir, causing a build-up of pressure. To meet physiological flow rates, an increased pressure drop is required.

Despite the increased peak pressure drop observed, our model predicts that the current range of distances that 

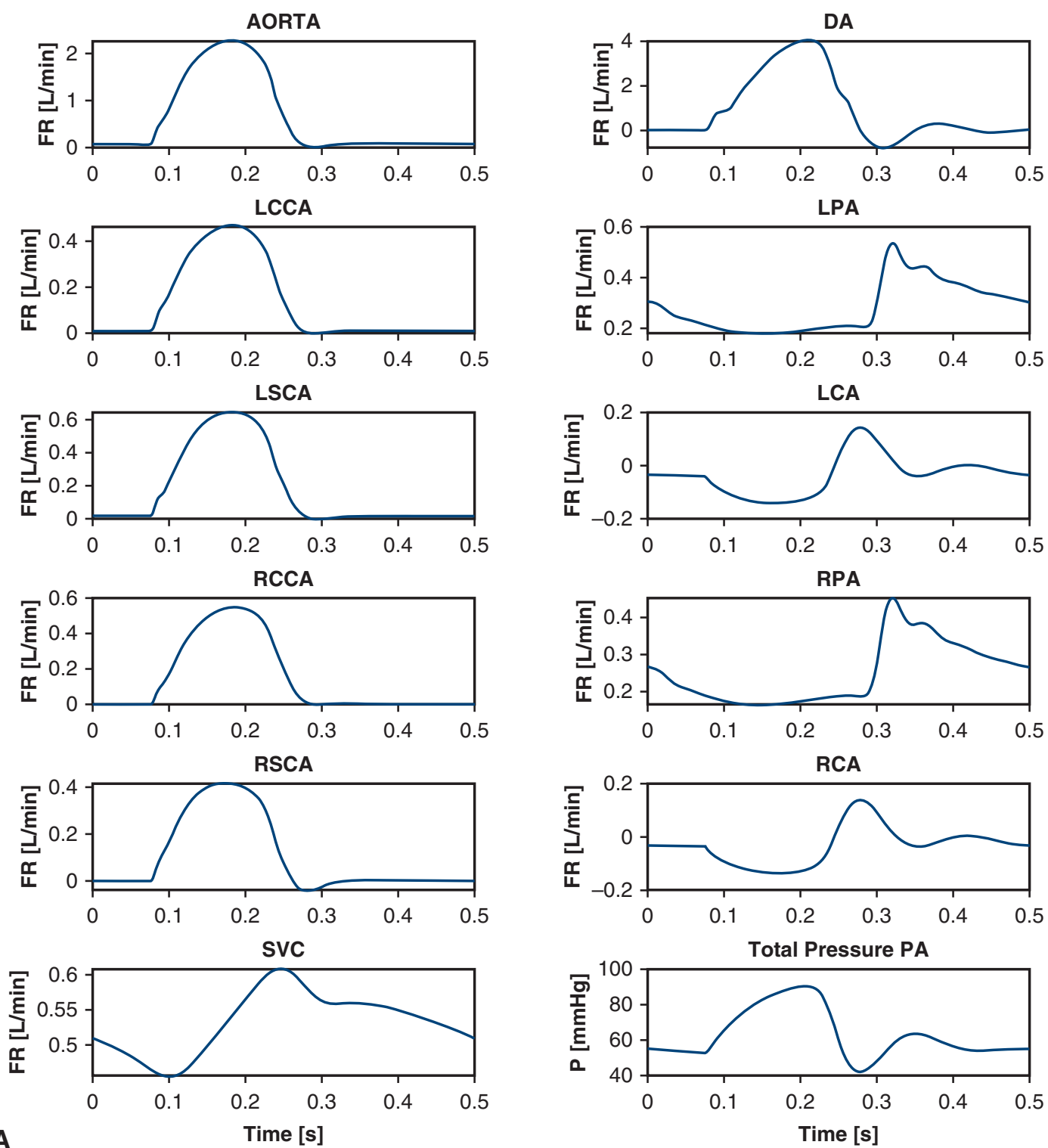

A

Time [s]

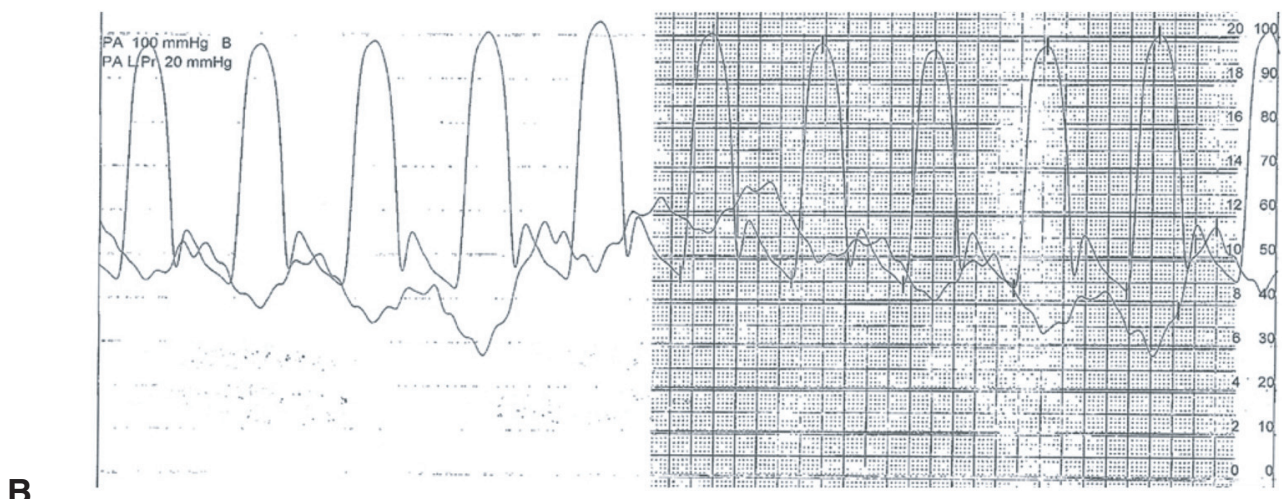

FIGURE 3. A, Sample boundary condition waveforms for all inlets and outlets (FR, flow rate; P, pressure). B, Main pulmonary artery and left pulmonary artery catheter pressure tracings in an infant after the HCS2 operation. $D A$, descending aorta; $L C C A$, left common carotid artery; $L P A$, left pulmonary artery; $L S C A$, left subclavian artery; $L C A$, left coronary artery; $R C C A$, right common carotid artery; $R P A$, right pulmonary artery; $R S C A$, right subclavian artery; $R C A$, right coronary artery; $S V C$, superior vena cava; $P A$, pulmonary artery/trunk. 


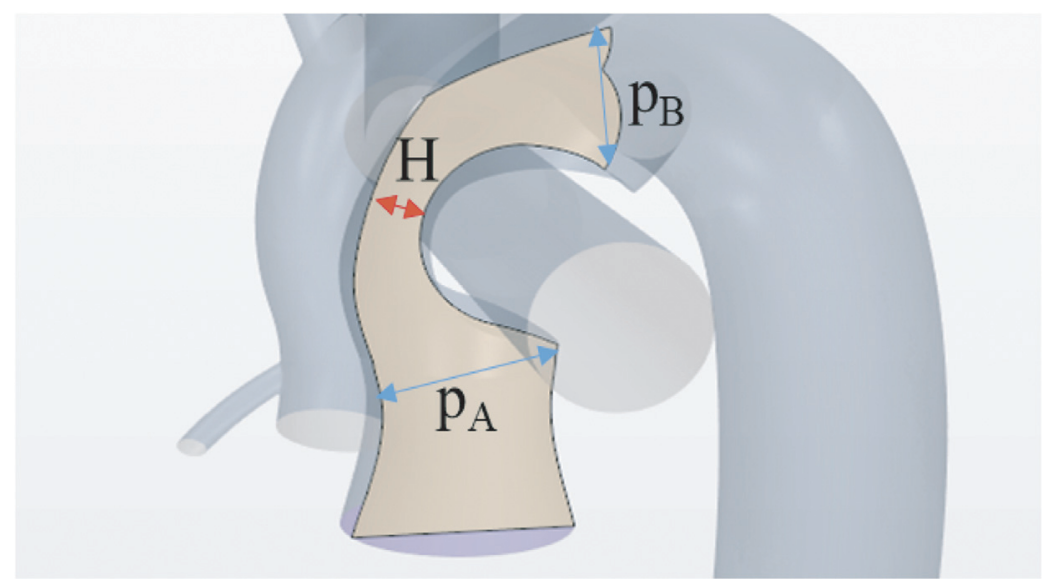

A

$\Delta p$ across Baffle
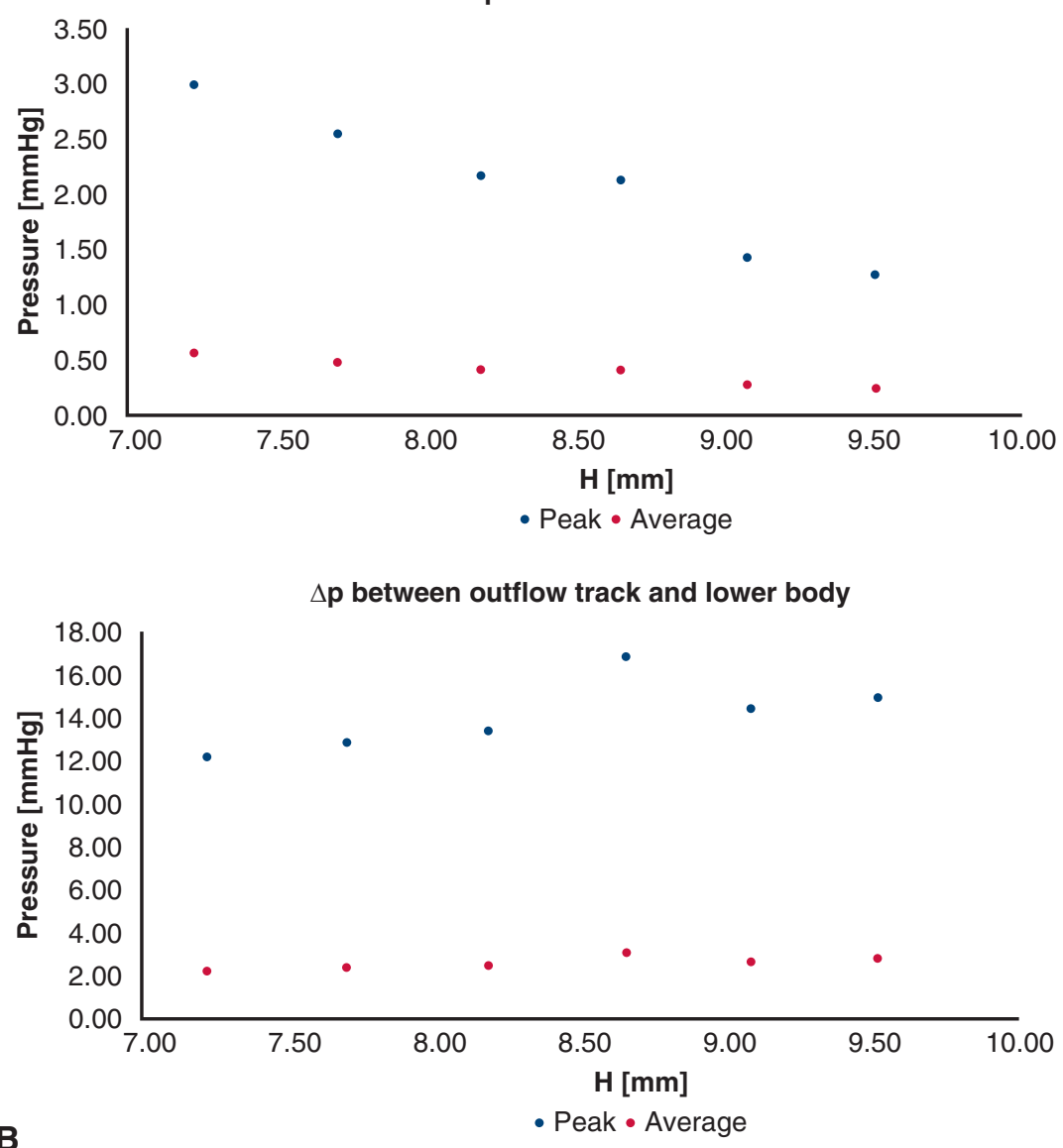

FIGURE 4. A, Location of the main pulmonary artery (MPA) lumen defining MPA narrowing characteristic height $(H)$. B, Peak and time-average, surfaceaveraged pressure drop across the baffle (top) and peak and time-averaged, surface-averaged pressure drop from baffle to distally to the ductal stent ( $(b o t t o m)$ versus the minimum distance between the anterior walls of the baffle and MPA. $P_{B}$, Distal baffle pressure; $P_{A}$, proximal baffle pressure.

we investigated falls within the predetermined clinical thresholds.

\section{Vortex Formation}

No baffle-related vortex shedding of significance was observed in our model, suggesting that vortex formation should not be of great concern. In the systemic circulation, the baffle can considered a half-cylinder in cross-flow. A feature of such flow is the von Kármán vortex street, caused by an unsteady flow separation around the cylinder. Flow vortices downstream from the baffle are undesirable, potentially leading to increased local thrombogenicity. The 
empirical relation in $\mathrm{Eq}$ (6), valid for an $R e$ range $250<\operatorname{Re}<2 \cdot 105$, is used to calculate the Strouhal number, $\left(S t=\frac{f D}{U}\right)$, a dimensionless number expressing the ratio of the frequency of vortex shedding, $f$, to main fluid velocity, $U$, for a given hydraulic diameter, $D$, characterizing the oscillatory nature of the flow,

$$
S t=0.198\left(1-\frac{19.7}{R e}\right)
$$

For the computed Reynolds number of $\mathrm{Re} \cong 1807$, the Strouhal number was found to be $\mathrm{St}=0.1958$. This corresponds to a shedding frequency of $\mathrm{f}=6.53 \mathrm{~Hz}$, or a characteristic formation time of 0.15 seconds. For a known shedding period and flow velocity, the distance required to form and sustain vortices can be calculated. Given an average fluid velocity of $0.5 \mathrm{~m} / \mathrm{s}$, the vortex transport distance is $\sim 75 \mathrm{~mm}$, far greater than the distance from the baffle to the aortic arch apex. This indicates a low probability of significant vortex propagation given the insufficient space for a vortex to fully form, as confirmed by the CFD model.

\section{Flow Field}

In this model, we study 3 separate circulatory regions: 2 high-pressure systems driven by the systemic ventricle and a low-pressure system in the pulmonary arteries. Figure 5 displays the unsteady velocity field predicted at 4 points during the heart cycle: late diastole, early systole, peak systole, and early diastole.

In the upper body systemic circulation, left ventricular output can be readily observed in systole, characteristic of the HCS2. In diastole, the upper body circulation experiences very mild retrograde flow owing to the presence of the coronary arteries. Flow stagnation is observed in the aortic arch apex as well. Coronary flow can be seen at its peak in early/mid-diastole (Figure 5, B, bottom), presenting as the characteristic out-of-phase waveform.

As expected, the pulmonary flow presents weak pulsatility compared with the systemic flow. Because of the $90^{\circ}$ T-junction shape of the SVC anastomosis to the pulmonary arteries, flow originating from the SVC impinges on the distal junction wall. However, owing to low flow velocities and lack of pulsatility in the pulmonary circulation, the branching flow does not present large recirculation or swirling.

As noted previously, the baffle can be thought of as a halfcylinder cut in cross-section. Figure 5, C provides further insight into the flow characteristics from the pulmonary trunk over the baffle. As the systolic phase picks up, the flow detaches from the baffle surface. In diastole, as the ventricular pressure gradient dissipates, the flow reattaches to the surface, following it all the way to the distal surface of the baffle. To elucidate the flow field across the MPA to the descending aorta (in combination with Figure 5, C), a sequence of cross-sectional velocity contour plots across a single heart cycle is provided in Figure E1.

Figure 6 shows the pressure gradient across the baffle. In diastole, owing to absence of right ventricle output and the anatomic enlargement of the MPA, the pulmonary root region experiences a large region of recirculation. The localized pressure increase in the upper section of the contour is caused by the converging-diverging nozzle effect induced by the spatially varying the anterior-posterior flow width in the MPA. This effect is most evident during diastole. These pressure fields can be partially responsible for the flow reattaching to the baffle surface.

\section{Baffle Loading and Wall Normal and Shear Stresses}

A main feature of the HCS2 is the presence of a baffle separating systemic and pulmonary flows; therefore, close inspection of the effects of the high-pressure flow on the baffle surface is important. The stent is subjected to uneven inward normal and shear loads throughout the cardiac cycle. Such uneven cyclic stresses could ultimately lead to mechanical failure. Figure 7 illustrates the wall shear stress (WSS) distribution on the baffle, as well as the locations of flow separation and reattachment on the baffle surface. It can be observed that in systole, shear stresses are high on the surface directly facing the right ventricle outlet, and, due to mild detachment, the WSS on the far sides drop to notably low values. Uneven contour distribution is due to the bend present in the ductal arch, which induces the typical swirling flow observed in normal arches. The secondary WSS peak observed in Figure 7, downstream of the baffle, is due to recirculation.

Figure 7, $A$ illustrates WSS and velocity to better understand where and how a diastolic WSS peak occurs at the distal end of the baffle. The peak can be seen to match the location where the flow reattaches to the surface. Although this peak is markedly smaller in magnitude compared with systolic peaks, this cyclic peak may induce some fatigue stress on the underlying tissue. The figure also illustrates the uneven distribution of the WSS during diastole that can be attributed to the geometries present in this model. Uneven stress distribution may have strong repercussions on how the endothelial tissue regenerates and heals following the procedure; however, the stent is expected to largely maintain its integrity. Structural analysis data (not reported here) appear to confirm this observation.

Figure 7, $B$ displays the WSS distribution on the baffle surface across one heart cycle; again the uneven distribution is ever-present. In addition, the WSS peaks appear to periodically migrate, leading to time-varying shear loads on the baffle. To further elucidate how the WSS distribution develops across a cycle with respect to the degree of MPA obstruction, we provide a more detailed representation in Figure E2, which compares the stented baffle loading for 
A
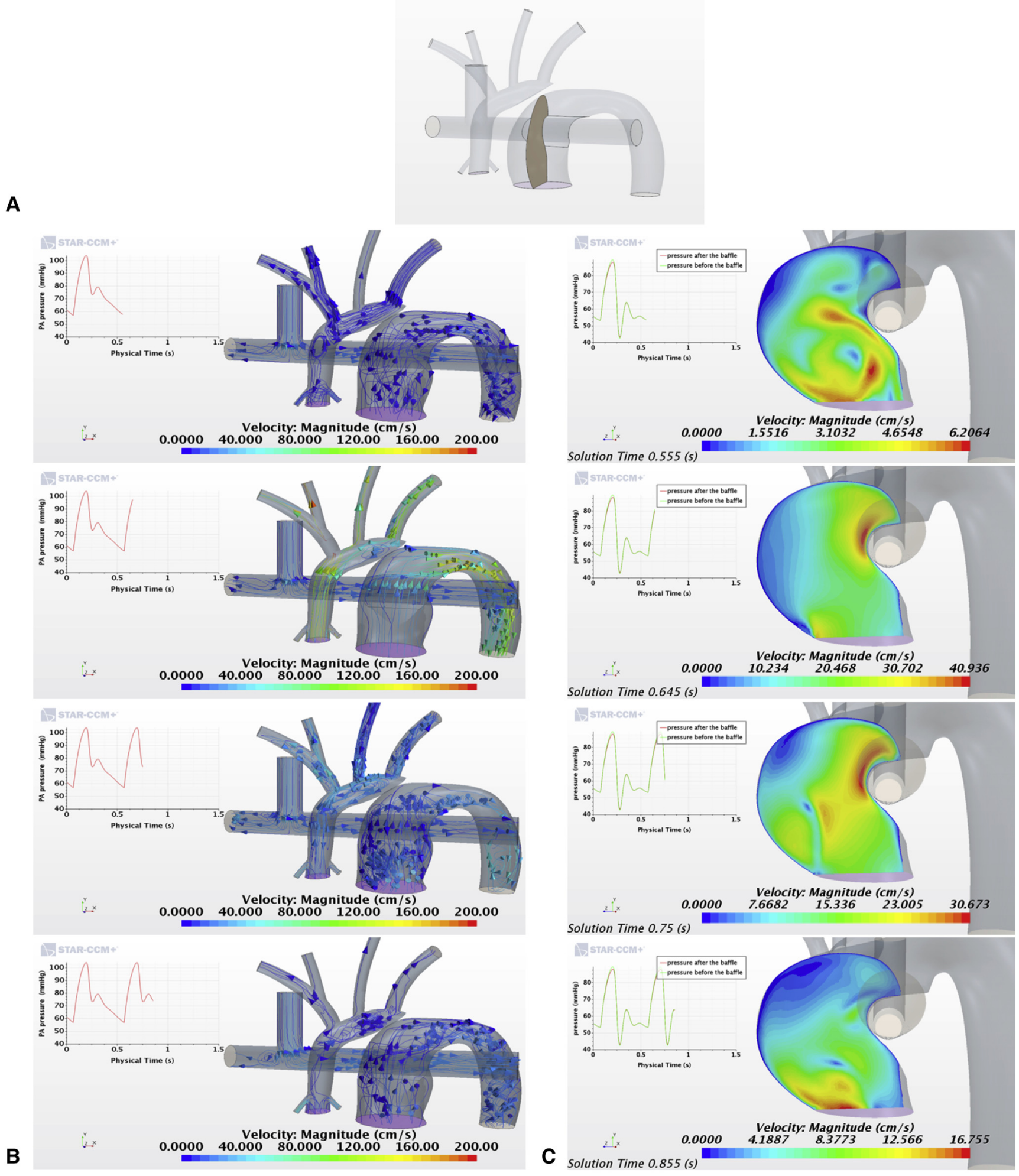

FIGURE 5. A, Anatomic plane of $\mathrm{C}$ and Figure 6 shown here as a shaded contour with the orientation of B. B and C, Velocity field representation using streamlines (B) and a pulmonary truck axial cross-sectional cut with a velocity field contour plot (C) for a second heart cycle. 

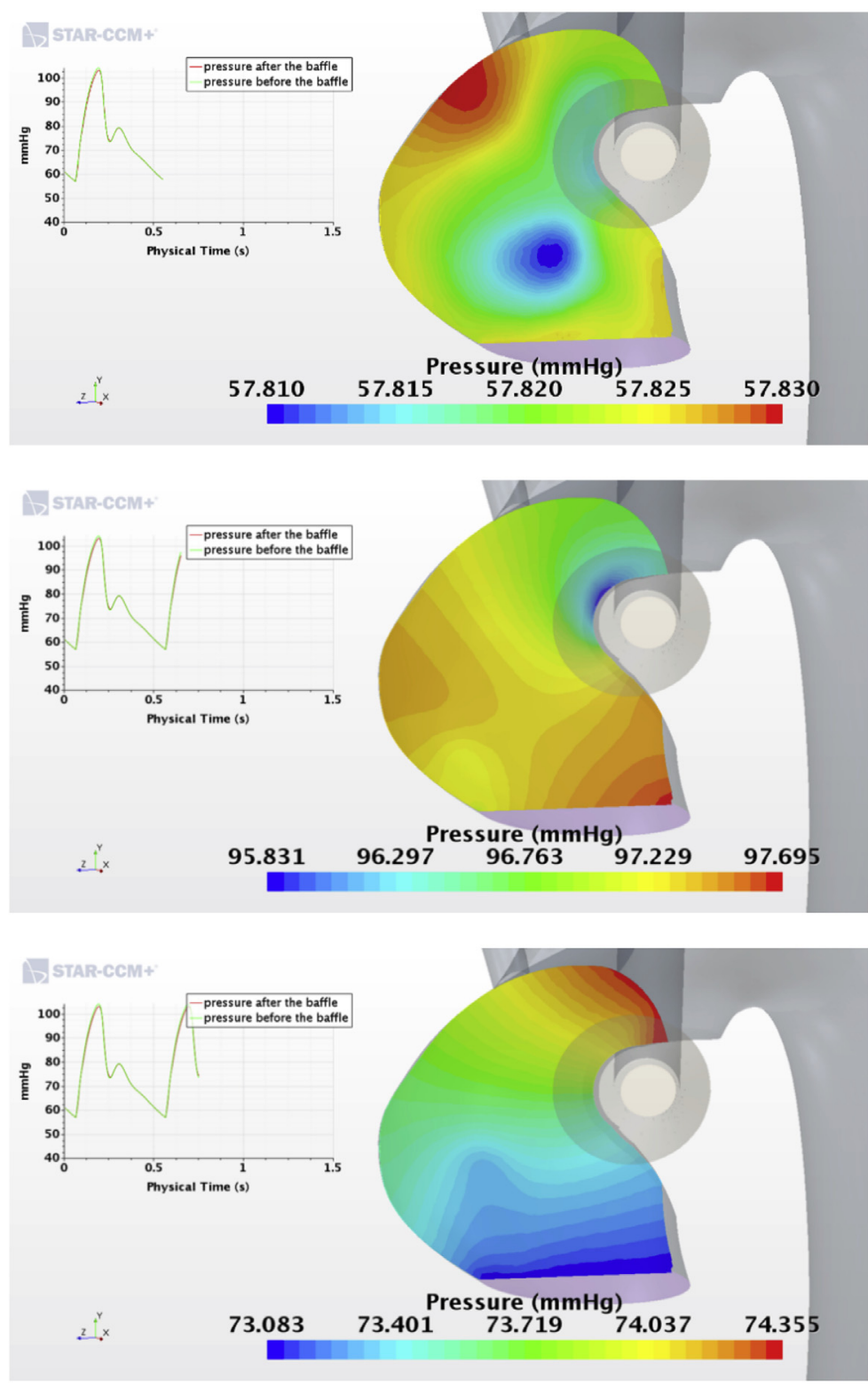

B

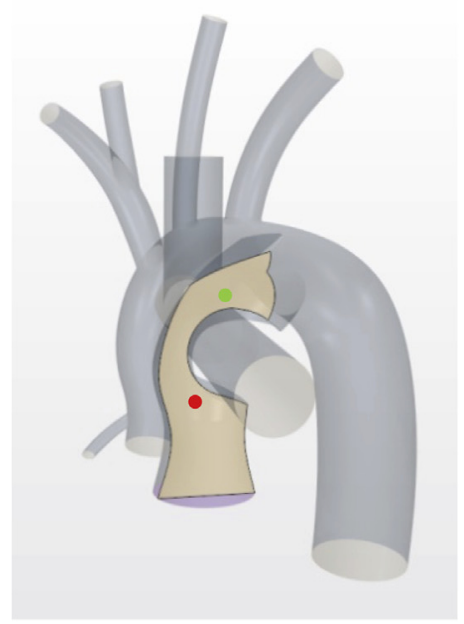

Pressure measurements locations
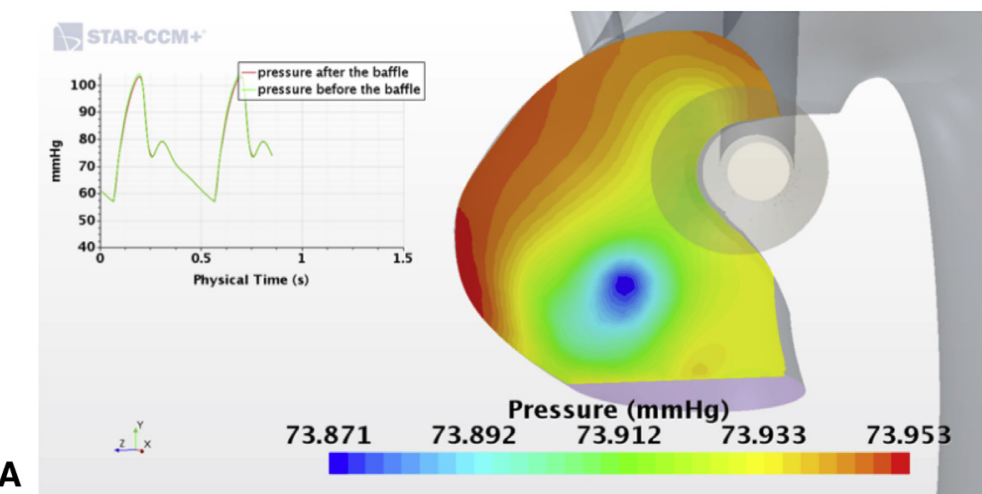

FIGURE 6. A, Pulmonary trunk axial cross-sectional cut with a pressure field contour plot. B, Pressure measurement locations. 

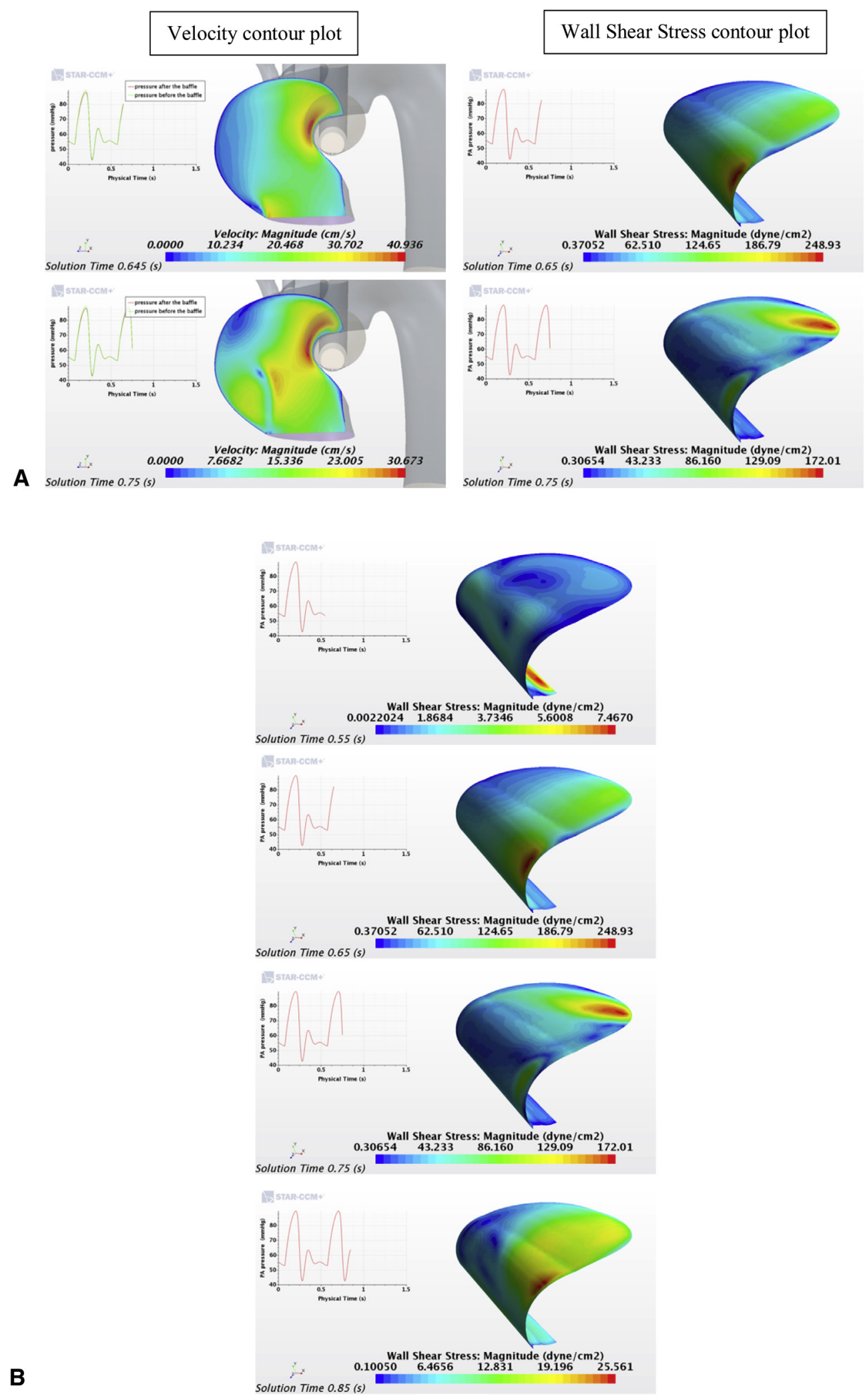

B

FIGURE 7. A, Velocity versus wall shear stress during early systole (top) and early diastole (bottom). B, Wall shear stress contour plot on the baffle surface in the systemic region. 
$2 \mathrm{H}$ values. The figure shows that the WSS magnitude and pattern do in fact change across a heart cycle.

\section{DISCUSSION}

In this study, we present a CFD analysis of a novel surgical procedure proposed for selected patients with SV CHD. Using CFD techniques, we investigated the flow in the systemic and pulmonary circulations. Under steady-state condition, we analyzed vortex shedding from the baffle and determined that, owing to inadequate distances, vortices would be unable to form. We then focused on geometrical features, such as converging-diverging nozzles, which produce flow accelerations and thus large, localized WSS loads. This was observed near the baffle. Large shear loads on the local tissue may cause vascular wall injury and lead to potential aneurysm formation. On the baffle, these loads may induce fatigue failure in the underlying stent. We will investigate these loading conditions and their long-term effects on the stent and on pulmonary blood flow in future work.

We isolated the baffle surface to further observe cyclic WSS loads. We have shown that throughout a cardiac cycle, the flow detaches in systole and subsequently reattaches to the baffle surface. This induces very distinct wall shear distributions on the surface. In systole, WSS magnitudes are elevated, and in diastole we observe uneven WSS distribution, which can alter the mechanical properties of the local tissue. It must be noted that owing to the complex topology, irregular flow patterns, such as recirculation regions and strong secondary flows, are generated. Given the current model, there is an opportunity to computationally explore, via shape optimization, topological improvements of the HCS2 to minimize these potentially pathologic flow patterns.

The effect of incremental flow obstruction due to the baffle was also investigated to ensure that the model did not include any MPA narrowing that could cause pressure drops across the baffle exceeding clinically suggested thresholds. We concluded that our geometries fell within the acceptable range.

This study has identified limitations in the rigid wall assumption applied to the entire fluid domain and the absence of actual modeled stents in the left pulmonary artery and in the ductus. The cyclic mechanical loads the outflow tract experiences can lead to a complex interaction between graft (noncompliant) and native tissue (compliant), which would alter the computed WSS pattern. In the distal arch, the ductal stent followed by the descending aorta results in an additional site of sharp compliance changes. The mechanical response of the stent to systemic loading has not been accounted for; this may become relevant over time as the geometry can be slowly altered, affecting the flow patterns observed.
The aim of this study was to elucidate possible initial problematic flow features in the proposed palliative treatment. The potential for obstruction to flow in the pulmonary trunk and the formation of vortices distal to the baffle has been shown to be minimal. The pressure gradient across the baffle is clinically acceptable, and no significant vortices were formed. These results show initial physiological flow fields and suggest that the HCS2 is a viable alternative for patients who qualify. Obviously, this study did not incorporate biological changes in the anatomy and physiology that could occur over time. A clinical update on the patients who have undergone this procedure is provided in an accompanying article in this issue of JTCVS Open. ${ }^{34}$

\section{Conflict of Interest Statement}

The authors reported no conflicts of interest.

The Journal policy requires editors and reviewers to disclose conflicts of interest and to decline handling or reviewing manuscripts for which they may have a conflict of interest. The editors and reviewers of this article have no conflicts of interest.

\section{References}

1. Fixler D, Nembhard W, Salemi J, Ethen M, Canfield M. Mortality in first 5 years in infants with functional single ventricle born in Texas, 1996 to 2003. Circulation. 2010;121:644-50.

2. Ohye R, Sleeper L, Mahony L, Newburger J, Pearson G, Lu M, et al. Comparison of shunt types in the Norwood procedure for single-ventricle lesions. New Engl J Med. 2010;362:1980-92.

3. Garcia Guerra G, Robertson C, Alton G, Joffe A, Dinu I, Nicholas D, et al. Quality of life 4 years after complex heart surgery in infancy. J Thorac Cardiovasc Surg. 2013;145:482-8.e2.

4. Dean P, Hillman D, McHugh K, Gutgesell H. Inpatient costs and charges for surgical treatment of hypoplastic left heart syndrome. Pediatrics. 2011;128: e1181-6.

5. Feinstein J, Woodrow Benson D, Dubin A, Cohen M, Maxey D, Mahle W, et al. Hypoplastic left heart syndrome: current considerations and expectations. J Am Coll Cardiol. 2012;59(1 Suppl):S1-42.

6. Ashburn D, McCrindle B, Tchervenkov C, Jacobs M, Lofland G, Bove E, et al. Outcomes after the Norwood operation in neonates with critical aortic stenosis or aortic valve atresia. J Thorac Cardiovasc Surg. 2003;125:1070-82.

7. Stanford Children's Health. Congenital heart disease. Available at: http://www. stanfordchildrens.org/en/topic/default?id=congenital-heart-disease-90-P02346. Accessed September 14, 2020.

8. Bacha E, Daves S, Hardin J, Abdulla R, Anderson J, Kahana M, et al. Singleventricle palliation for high-risk neonates: the emergence of an alternative hybrid stage I strategy. J Thorac Cardiovasc Surg. 2006;131:163-71.e2.

9. Venugopal P, Luna K, Anderson D, Austin C, Rosenthal E, Krasemann T, et al. Hybrid procedure as an alternative to surgical palliation of high-risk infants with hypoplastic left heart syndrome and its variants. J Thorac Cardiovasc Surg. 2010;139:1211-5.

10. Galantowicz M, Cheatham J. Lessons learned from the development of a new hybrid strategy for the management of hypoplastic left heart syndrome. Pediatr Cardiol. 2005;26:190-9.

11. Galantowicz M, Cheatham J, Phillips A, Cua C, Hoffman T, Hill S, et al. Hybrid approach for hypoplastic left heart syndrome: intermediate results after the learning curve. Ann Thorac Surg. 2008;85:2063-71.

12. Baba K, Kotani Y, Chetan D, Chaturvedi R, Lee K, Benson L, et al. Hybrid versus Norwood strategies for single-ventricle palliation. Circulation. 2012;126(11 Suppl 1):S123-31.

13. DeCampli W, Fleishman C, Nykanen D. Hybrid approach to the comprehensive stage II operation in a subset of single-ventricle variants. J Thorac Cardiovasc Surg. 2015;149:1095-100. 
14. Nationwide Children's. Hypoplastic left heart syndrome: overview and hybrid treatment. Available at: http://www.nationwidechildrens.org/hypoplastic-leftheart-syndrome. Accessed September 14, 2020.

15. Caldarone C, Benson L, Holtby H, Van Arsdell G. Main pulmonary artery to innominate artery shunt during hybrid palliation of hypoplastic left heart syndrome. J Thorac Cardiovasc Surg. 2005;130:e1-2.

16. Ceballos A, Argueta-Morales I, Divo E, Osorio R, Caldarone C, Kassab A, et al. Computational analysis of hybrid Norwood circulation with distal aortic arch obstruction and reverse Blalock-Taussig shunt. Ann Thorac Surg. 2012;94: 1540-50.

17. Ceballos A, Blanchette L, Argueta-Morales IR, Divo EA, Kassab AJ, DeCampli WM. Hemodynamic analysis of the hybrid Norwood procedure for hypoplastic left heart syndrome using a coupled CFD-lumped parameter model [abstract]. In: Proceedings of the 4th International Conference on Computational and Mathematical Biomedical Engineering, Cachan, France, June 29 to July 1; 2015.

18. Ceballos A, Prather R, Divo E, Kassab A, DeCampli W. Patient-specific multiscale model analysis of hemodynamics following the hybrid Norwood procedure for hypoplastic left heart syndrome: effects of reverse Blalock-Taussig shunt diameter. Cardiovasc Eng Technol. 2018;10:136-54.

19. Hsia T, Cosentino D, Corsini C, Pennati G, Dubini G, Migliavacca F. Use of mathematical modeling to compare and predict hemodynamic effects between hybrid and surgical Norwood palliations for hypoplastic left heart syndrome. Circulation. 2011;124(11 Suppl 1):S204-10.

20. Baker C, Corsini C, Cosentino D, Dubini G, Pennati G, Migliavacca F, et al. Effects of pulmonary artery banding and retrograde aortic arch obstruction on the hybrid palliation of hypoplastic left heart syndrome. J Thorac Cardiovasc Surg. 2013;146:1341-8.

21. Corsini C, Biglino G, Schievano S, Hsia T, Migliavacca F, Pennati G, et al. The effect of modified Blalock Taussig shunt size and coarctation severity on coronary perfusion after the Norwood operation. Ann Thorac Surg. 2014;98:648-54.

22. Liu J, Un Q, Hong H, Sun Y, Liu J, Qian Y, et al. Medical image-based hemodynamic analysis for modified Blalock-Taussig shunt. J Mech Med Biol. 2015;15: 1550035 .

23. Esmaily Moghadam M, Migliavacca F, Vignon-Clementel I, Hsia T, Marsden A. Optimization of shunt placement for the Norwood surgery using multi-domain modeling. J Biomech Eng. 2012;134:051002.
24. Good B, Deutsch S, Manning K. Hemodynamics in a pediatric ascending aorta using a viscoelastic pediatric blood model. Ann Biomed Eng. 2015;44:1019-35.

25. Fox R, McDonald A, Pritchard P, Mitchell J. Fluid Mechanics. Hoboken, NJ: Wiley; 2016.

26. Bove E, Migliavacca F, de Leval M, Balossino R, Pennati G, Lloyd T, et al. Use of mathematic modeling to compare and predict hemodynamic effects of the modified Blalock-Taussig and right ventricle-pulmonary artery shunts for hypoplastic left heart syndrome. J Thorac Cardiovasc Surg. 2008;136:312-20.e2.

27. Pennati G, Migliavacca F, Dubini G, Pietrabissa R, de Leval M. A mathematical model of circulation in the presence of the bidirectional cavopulmonary anastomosis in children with a univentricular heart. Med Eng Phys. 1997;19:223-34.

28. Pennati G, Migliavacca F, Dubini G, Pietrabissa R, Fumero R, de Leval M. Use of mathematical model to predict hemodynamics in cavopulmonary anastomosis with persistent forward flow. J Surg Res. 2000;89:43-52.

29. Paeme S, Moorhead K, Chase J, Lambermont B, Kolh P, D'orio V, et al. Mathematical multi-scale model of the cardiovascular system including mitral valve dynamics. Application to ischemic mitral insufficiency. Biomed Eng Online. 2011;10:86.

30. Jalali A, Jones G, Licht D, Nataraj C. Application of mathematical modeling for simulation and analysis of hypoplastic left heart syndrome (HLHS) in pre- and postsurgery conditions. Biomed Res Int. 2015;2015:1-14.

31. Prather R, Kassab A, Ni M, Divo E, Argueta-Morales R, DeCampli W. Multiscale pulsatile CFD modeling of thrombus transport in a patient-specific LVAD implantation. Int J Numer Methods Heat Fluid Flow. 2017;27:1022-39.

32. Faragallah G, Wang Y, Divo E, Simaan M. A new control system for left ventricular assist devices based on patient-specific physiological demand. Inverse Probl Sci Eng. 2012;20:721-34.

33. Sankaran S, Esmaily Moghadam M, Kahn A, Tseng E, Guccione J, Marsden A. Patient-specific multiscale modeling of blood flow for coronary artery bypass graft surgery. Ann Biomed Eng. 2012;40:2228-42.

34. Farias M, Fleishman CE, Nykanen D, DeCampli WM. Clinical update on the hybrid comprehensive stage II operation. JTCVS Open. 2021;7:327-35.

Key Words: computational fluid dynamics, lumped parameter model, hypoplastic left heart syndrome, congenital heart disease 


\section{APPENDIX E1}

\section{Loss Coefficient}

Based on Bernoulli's equation, the mass flow rate across the orifice is

$$
\dot{m}=\frac{K A_{V S D}}{\sqrt{1+\beta^{4}}} \sqrt{2 \rho \Delta P_{V S D}}
$$

where $\beta$ is the diameter ratio of the orifice to the nominal right ventricular diameter, $\rho$ is the fluid density, $K$ is a loss coefficient, and $A_{V S D}$ and $\Delta P_{V S D}$ are the area of the orifice and the pressure difference across the defect, respectively. ${ }^{22}$ The loss coefficient for an orifice can be obtained by curve fitting of empirical data as

$$
K=0.5959+0.0312 \beta^{2.1}-0.184 \beta^{8}+\frac{91.71 \beta^{2.5}}{R e_{D_{V}}^{0.75}}
$$

where $R e_{D_{V}}$ is the Reynolds number based on the right ventricle nominal diameter. ${ }^{32}$ The loss coefficient expression can be further simplified given that $\beta$ is small $(<1)$, leading Eq A.2 to be reduced to $K=0.5959$.

\section{LPM Equations and Elastance Function}

$$
\Delta p=L \frac{d Q}{d t}+R Q
$$

$$
Q=C \frac{d(\Delta p)}{d t}
$$

Equations A.3 and A.4 are the equivalent Kirchhoff node and loop laws; here $Q$ is the volume flow rate and $\Delta p$ is the pressure difference between two subsequent nodes.

The "double hill" normalized time-dependent elastance function, $E(t)=\left[E_{\max }-E_{\min }\right] E_{n}\left(t_{n}\right)+E_{\min }$, is used to model the right ventricle ${ }^{28,29}$ and to drive the LPM,

$$
E_{n}\left(t_{n}\right)=\left[\frac{\left(\frac{t}{\frac{\frac{T}{m a x}}{0.303}}\right)^{1.32}}{1+\left(\frac{t}{\frac{T}{\max }}\right)^{1.32}}\right]\left[\frac{1}{\left.1+\left(\frac{t}{\frac{T}{\max }}\right)^{2.508}\right)^{21.9}}\right]
$$

Here $t_{n}=\frac{t}{T}$ is the normalized time with Tmax $=0.2+0.15 t_{c}$ and $t_{c}=\frac{60}{H R}$, where $H R$ is the heart rate. 

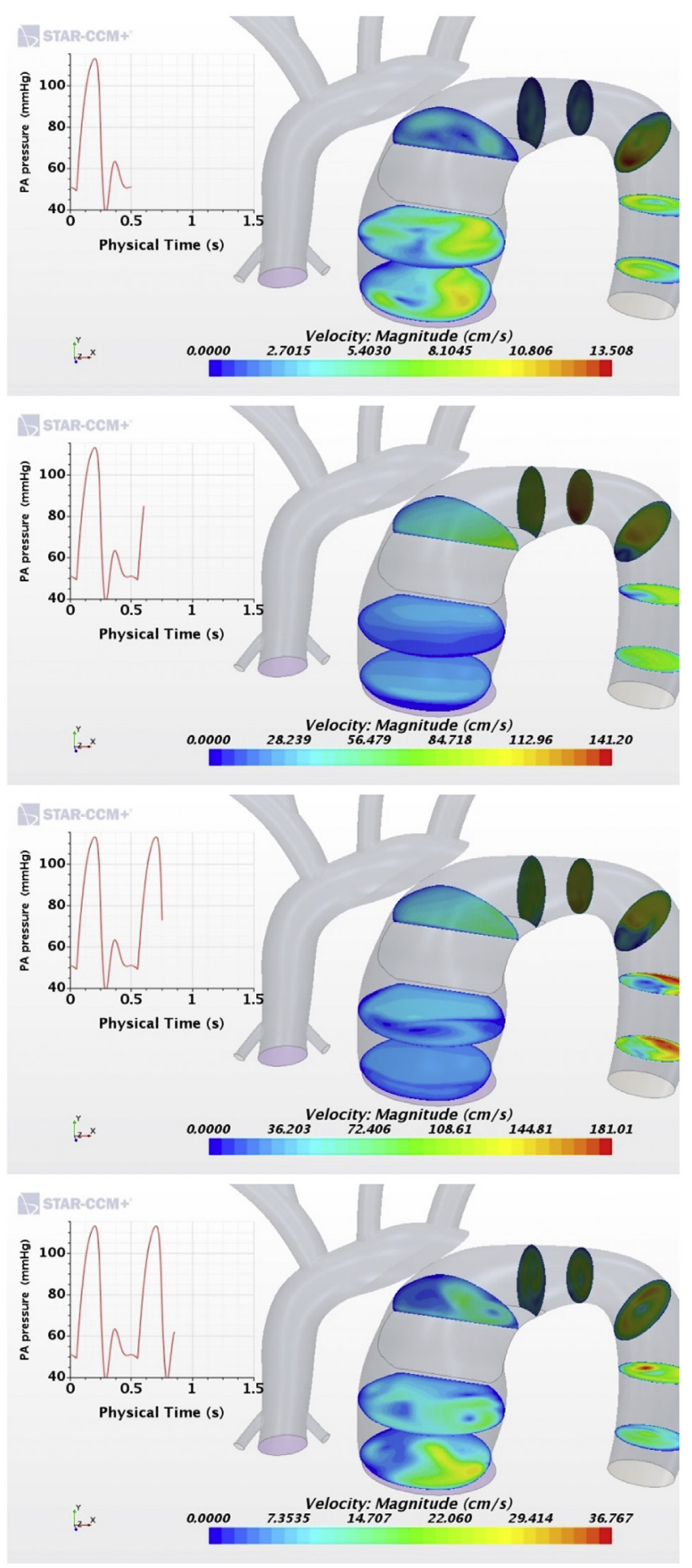

FIGURE E1. Sample cross-sectional velocity contour plots across one heart cycle for the MPA, distal arch and DA. 

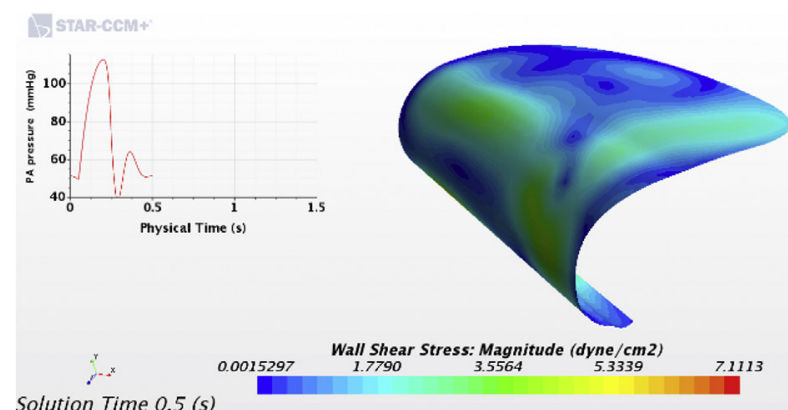

Solution Time $0.5(\mathrm{~s})$
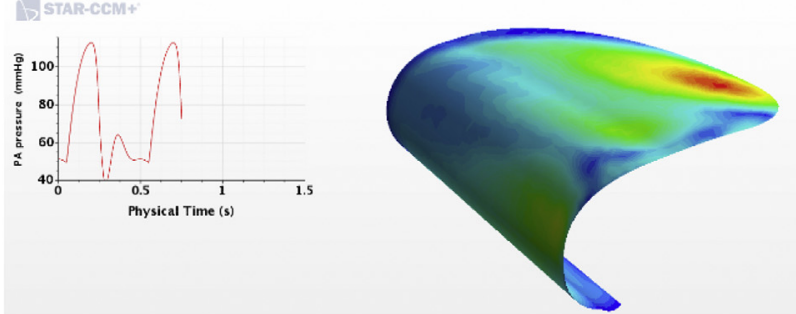

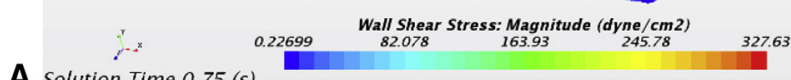
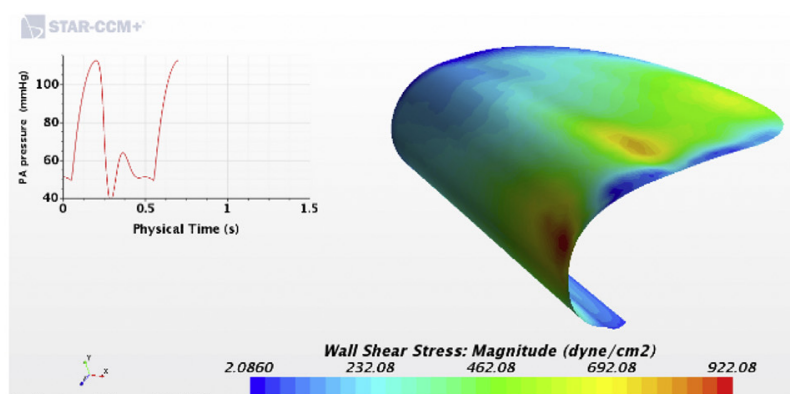

Wall Shear Stress: Magnitude (dyne/cm2) Solution Time $0.7(s)$
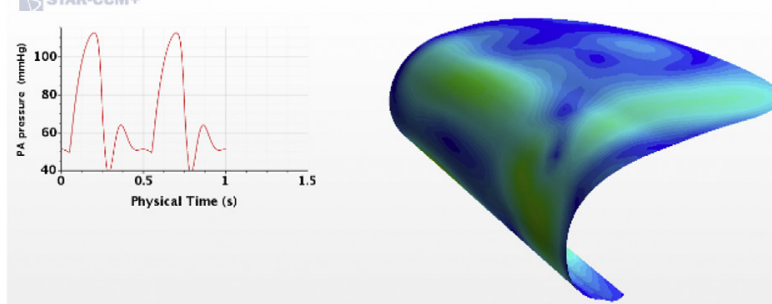

1. Wall Shear Stress: Magnitude (dyne/cm2) Solution Time $1(s)$
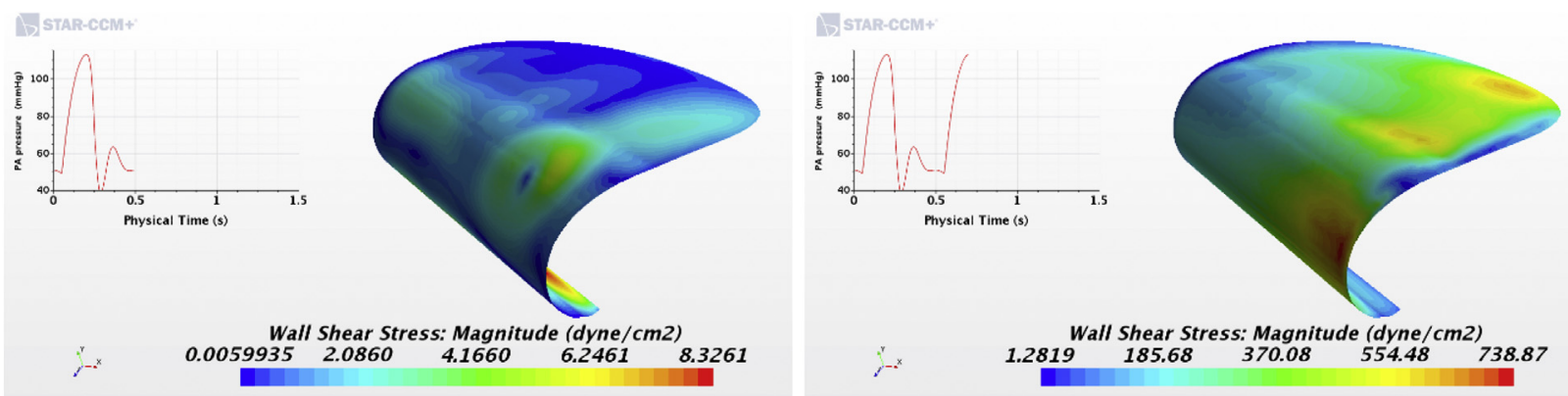

Wall Shear Stress: Magnitude (dyne/cm2)

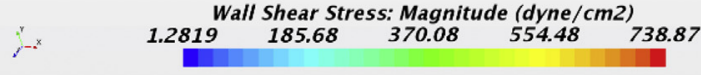

STAR-CCM
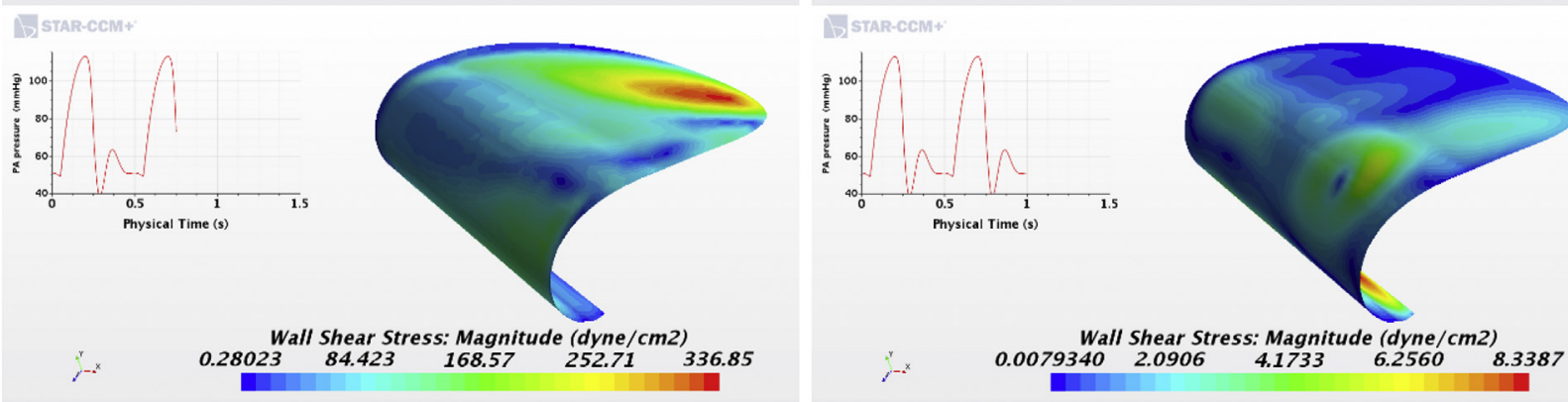

FIGURE E2. Comparison of stented baffle WSS for two degrees of MPA obstruction, (A) $\mathrm{H}=7.71 \mathrm{~mm}$ and (B) $\mathrm{H}=8.19 \mathrm{~mm}$. 